\title{
A CASE STUDY ON SIMULATION AND EMULATION OF A NEW CASE PICKING SYSTEM FOR A US BASED WHOLESALER
}

\author{
Ralf Bleifuß \\ SSI Schaefer Noell GmbH \\ Klingholz 18/19 \\ D-97232 Giebelstadt, GERMANY
}

\author{
Sven Spieckermann \\ SimPlan AG \\ Edmund-Seng-Straße 3-5 \\ D-63477 Maintal, GERMANY
}

\author{
Stephan Stauber \\ SimPlan AG \\ Bruderwöhrdstraße 15b \\ D-93055 Regensburg, GERMANY
}

\begin{abstract}
This paper presents a comprehensive and long-term joint simulation project in the area of warehouse logistics. The project comprised six stages with the first three stages being part of the development and evaluation of a new storage and picking technology. This technology is a specific case picking approach and it includes process steps like storage of product pallets, automated de-palletizing, storage of product layers, the separation of product cases from layers, sequencing and palletizing of product cases. Within the second part of the project the technology was adapted to the requirements of a US-based wholesaler. The simulation activities within this second part started with classical planning simulation and covered the emulation of the real-world control software, the support of the system ramp-up, and finally the implementation of a permanent test base in order to evaluate necessary software changes. The article describes the storage and picking technology, the stages of the simulation project and the benefits of both, the technology itself and the extensive simulation application.
\end{abstract}

\section{INTRODUCTION}

Design and operation of warehouses have been subject to numerous research activities and case studies as the comprehensive surveys by Gu, Goetschalckx, and McGinnis (2010), Rouwenhorst et al. (2000), and Roodbergen and Vis (2009) are showing. Amongst the approaches to tackle the challenges associated with warehouse design and operation, discrete-event simulation (DES) is playing a vital role. Together with analytic methods (based on optimization techniques) and heuristic methods (based on rules and experience) Ashayeri and Gelders (1985) list DES as one of the three methods of choice when it comes to the evaluation of warehouse equipment. Discussing performance evaluation of warehouses $\mathrm{Gu}$, Goetschalckx, and McGinnis (2010) put DES in one row with analytical models and benchmarking (comparison with the performance of other warehouses). A systematic collection and comparison of warehouse design approaches presented by Baker and Canessa (2009) also highlights the importance of DES. Smith (2003) denotes in his survey on the use of DES, that material handling system design always has been an "extremely popular area for the application of simulation", and warehouse systems typically comprise plenty of material handling operations. 


\section{Bleifuß, Spieckermann, and Stauber}

Based on this background it is no surprise that large material handling equipment suppliers typically rely on the application of DES within their projects as well. This paper presents a case study around a project of the equipment and solution supplier SSI Schaefer Noell, who had to design, deliver, install and ramp-up a distribution center for a US-based wholesaler. DES was involved in the system design quite early, however, the application of simulation was not limited to support design decisions but it was extended to test real-world control software like the WMS (Warehouse Management System) and the MFC (Material Flow Control), a methodology known as emulation, virtual ramp-up, or soft commissioning (Auinger, Vorderwinkler, and Buchtela 1999; Johnstone, Creighton, and Nahavandi 2007). Figure 1 is giving an overview on the project phases and the use and benefits of DES in the respective phase.

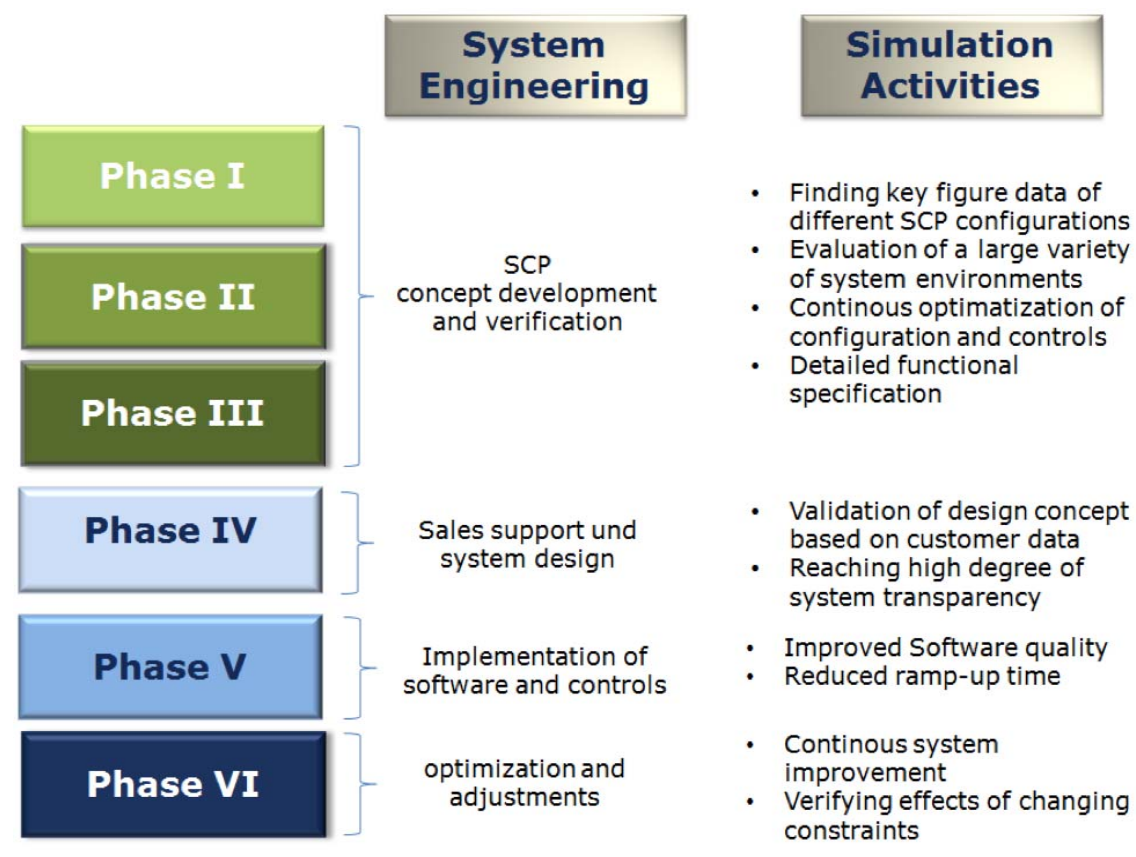

Figure 1: Project phases and simulation objectives

Basically, the outline of this paper is following these phases. However, since one challenge in the project was that a new material handling technology was going to be deployed for the first time, the technology had to be tested in itself and in the specific application context of the wholesaler. Hence, the following section introduces this so-called case picking technology after a brief description of distribution center processes in general and a short introduction to case picking. As the considered picking technology is quite complex and as its understanding is essential for the understanding of the challenges on the simulation side, Section 2 is taking up some space. Section 3 will give an overview on the simulation work during the design of the new material handling concept. Section 4 is focused on the simulation activities during the adaptation of the concept for the wholesaling company and gives some insight in the emulation of the systems control logic which has been conducted in addition to the design simulation within this project. Section 6 briefly summarizes the paper.

\section{CASE PICKING}

In order to understand the simulated warehouse system, it is helpful to understand the processes in distribution centers in general and to understand the specific need for and challenges of automated case picking. Both will be discussed in the next two subsections prior to a discussion of the specific solution implemented for the wholesaling company. 


\section{Bleifuß, Spieckermann, and Stauber}

\subsection{Distribution Center Processes}

In order to understand the processes in distribution centers two terms need to be introduced first: SKU (stock keeping unit) and (customer) order. An SKU is a unique identifier for a specific product within a specific packaging. For example, in a warehouse storing beverages, all half-liter bottles of soda would have one SKU while all liter bottles of the same soda would have different SKU. Orders are placed by customers comprise various quantities of one or more SKUs. The distribution center has to process these orders, i.e. it has to ship the ordered SKUs to the respective customer. In order to be able to do so, there are the following main steps which are common more or less for all distribution centers:

- the receiving process which collects products coming in from suppliers of the distribution center,

- the storage process which stores the received products in designated storage areas. Note, that in general there might be more than one storage area depending on the size of the products and because of a structuring of the storage procedures in several levels (as will be illustrated by the SCP example in Section 2.3),

- the picking process where SKUs are retrieved from their storage areas and are taken to the subsequent process step which is

- the sorting or consolidation process where products belonging to the same customer order are collected, and

- the shipping area where the orders are leaving the distribution centers and are shipped to the customer

A short characterization of the processes (which is presented in a similar way by, e.g., Rouwenhorst et al. 2000) necessarily leads to generalization. There of course are warehouses without any consolidation area or where the shipping area is serving at the same time as sorting space. In some distribution centers, there is no consolidation of orders but of shipments comprising several orders. And, in a warehouse used for example as part of a manufacturing process of one company, suppliers and customers might all reside under the same firm (but in this case are at least different departments). And, on top of the listed processes, there also might be additional tasks to cover, e.g., related to quality with respect to received or shipped products. The purpose of this description, however, is not to cover all possible process topologies of warehouses but to give an brief overview on the typical main process steps.

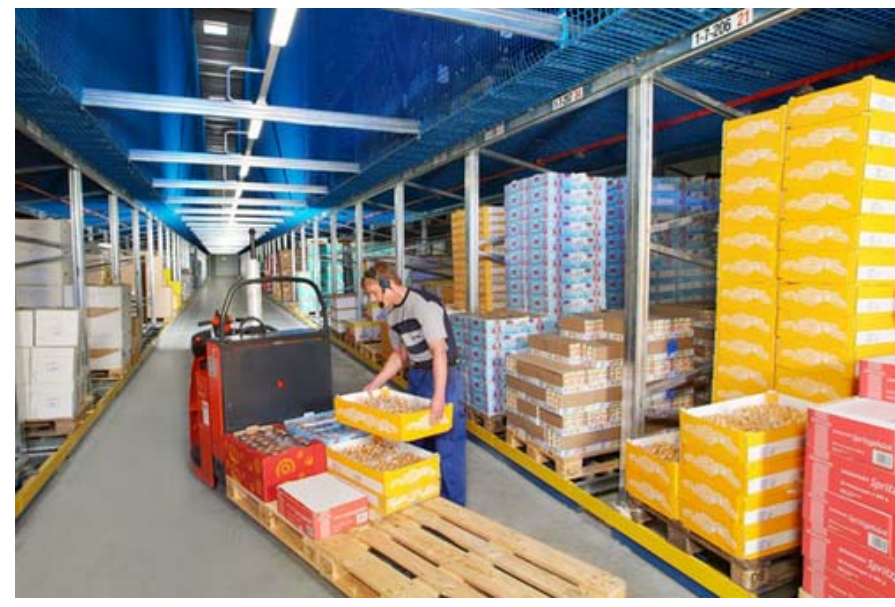

Figure 2: Example of a full case picking process

\subsection{Automated Case Picking}

There are different ways to handle the order picking process with respect to the packaging of the received and picked products. The case considered in this article will focus on incoming pallets each carrying cases with one SKU or so-called rainbow pallets with one SKU per layer. The picking process is focused on socalled full case picking, i.e. full cases of one SKU are retrieved from their storage location and are moved 


\section{Bleifuß, Spieckermann, and Stauber}

to the next process step. Figure 2 shows an example of a picking and storage area with single SKUs stored on pallets and a manual full case picking process using a pallet truck.

For several reasons (such as growing labor costs, high demands on ergonomics, increasing transportation costs and pressure on operations for reducing damage in transit), there is increasing demand to implement automated ways of case picking. Gilmore and Holste (2009) present an in-depth discussion of the motivation to invest in picking automation and an comprehensive overview on a variety of automated solutions. One of these solutions, the SCP, has been simulated as part of this case study. It is introduced in the next section.

\subsection{The SCP Solution}

The SCP (Schaefer Case Picking) solution covers all of the processes of a distribution center as discussed in Section 2.1 (see also the illustration in Figure 3):

1. There is a pallet storage area collecting the pallets being delivered from suppliers. Each of the pallets carries several layers of cases of a single SKU.

2. The second process step takes (mainly automatic) single layers from pallets and puts each layer on a tray.

3. These trays carrying single layers of cases (again of course of one SKU) are put in a tray warehouse (which again is a storage area). This tray warehouse is called STS (Schaefer Tray System), where multiple AS/RS devices (automated storage and retrieval device) can be arranged vertically.

4. Whenever an SKU is requested by a customer order, the tray carrying the SKU is retrieved from the tray storage and moved by an AS/RS device to a so called case-wheeler lift where a sophisticated conveyor mechanism combined with special vision technology is automatically picking one or more case down from the tray onto an intermediate buffer. The cases sitting on the intermediate buffer are feeding into collecting conveyor belts. The combination of case-wheeler lifts, STS with AS/RS devices, and assorted collecting conveyors is called SCP module.

5. The picking process by the case-wheeler lifts and the feeding into the collecting conveyors needs to be organized such that the cases are picked according to pre-calculated sequences. The calculation of each of these sequences is based upon the customer orders (which may need to be splitted into several pallets), information related to the target store (which is called "store readiness" and tries to account for the arrangement of the SKUs on the shelves in the customer store), information on weight and measurement of the cases (because the packing density as well as some stability criteria have to be taken into account), and on some SKU specific constraints related to material etc. The calculation is performed by a software package called SPPG (Schaefer Pack Pattern Generator). The whole picking process starting at the STS and ending on the collection conveyors needs to be controlled in such a way that the cases are finally in sequence on the conveyors, however, when the pallets structure allows the building of sequence groups, the sequence within a group can dynamically be rearranged.

6. Each collecting conveyor is moving the cases to a dedicated automatic palletizer stacking the cases on a customer order pallet. After wrapping and labeling, the pallet is ready to be shipped.

Several questions were arising when SSI first developed ideas for the SCP:

- Will the collecting conveyors be able to continuously supply the automatic palletizers?

- Will the case-wheeler lifts be able to make sure that enough cases are fed into the collecting conveyors in sequence?

- Will the AS/RS devices of the STS be able to move enough pallets to the case-wheeler lifts and to store them again (after the required cases have been picked)?

- Will the overall system from full pallet arrival to customer pallet shipping work as expected? 


\section{Bleifuß, Spieckermann, and Stauber}

In order to answer these questions, SSI initiated a simulation study which was conducted in parallel to engineering and design steps of the SCP. Part of the simulation activities was not only to evaluate the material handling elements but also to refine (and at some points actually to define) the control rules for the SCP components which had to be designed in a way that it could account for parameters like number of cases per order line (called "wheel factor" with respect to the case-wheeler operation), the number and frequency of SKUs, the height of the cases to be stored, the structures of the customer pallets, the replenishment and some other factors.
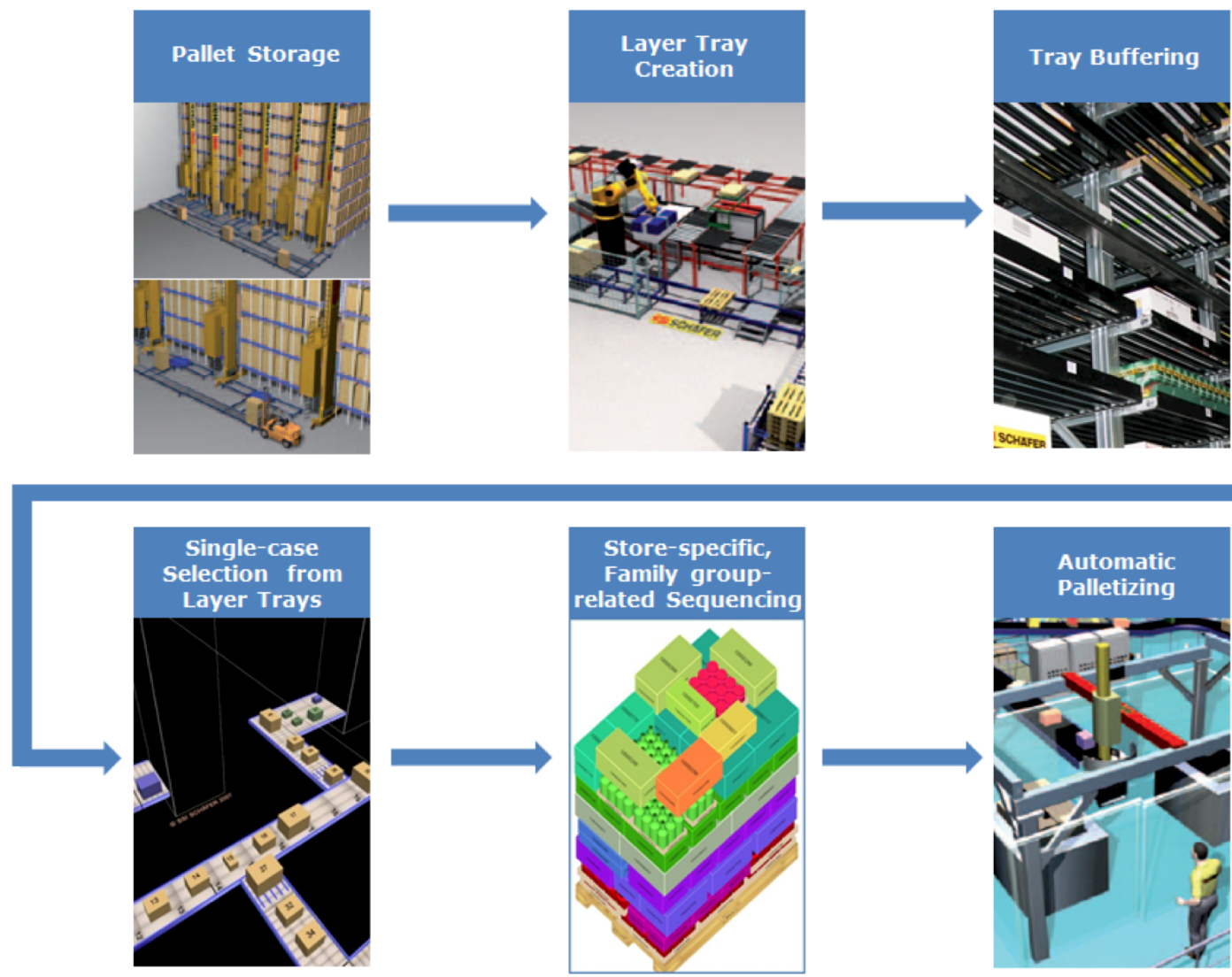

Figure 3: Process steps of the SCP system

\section{SIMULATION FOR SCP DESIGN}

The simulation activities supported the SCP design basically following the questions outlined in the preceding section. This section is organized accordingly, however, the simulation activities of the first two questions are discussed in one subsection. The software platform for the simulation and emulation activities was Plant Simulation from Siemens (Bangsow 2010), the simple reason being that it is the standard tool for material handling simulations at SSI Schaefer for many years now. To model complex warehouses, there is a library called "Logistics Suite" for Plant Simulation. It provides different types of conveyors, a generic and configurable material flow control, and many more building blocks which enable an efficient modeling.

\subsection{Simulation of collecting belts, case-wheeler lifts, and attached elements}

In order to fully describe the challenge in this first phase of simulation activities it is necessary to consider the material flow of the cases between STS and customer pallet in a little more detail. Figure 4 shows an schematic illustration of these material handling components and Figure 5 shows a schematic 2Dsimulation layout where the levels of the tray storage are tilted to the left and right hand side (for the left 


\section{Bleifuß, Spieckermann, and Stauber}

and the right tray storage, respectively), the connecting conveyors are displayed side by side in the middle of the drawing, and the case-wheeler lifts are drawn horizontally across the tilted tray storage racks.

The way of a case from the tray stored in an STS down to a collection conveyor is as follows: the tray is retrieved from one of the AS/RS devices in the tray storage (taking the left hand and the right hand side of the SCP module together with $2 \cdot 5$ levels there are $10 \mathrm{AS} / \mathrm{RS}$ devices within one SCP module). Since the AS/RS device cannot hand over the tray to a case-wheeler lift directly, it is stored on one of the transfer locations. The case-wheeler lift is collecting the tray at the transfer location and is moving it to an intermediate buffer belt. There, the required cases are picked from the tray (using a specific wheeling mechanism, hence the name of the lifts) and are conveyed onto the intermediate buffer. Note that each collecting conveyor has one dedicated intermediate buffer for each case-wheeler lift leading to 14 intermediate buffers per collecting conveyor for the seven left hand and seven right hand case-wheeler lifts depicted in Figure 5. A case remains on an intermediate buffer until it can be inserted to the collection conveyor according to the pre-calculated sequence information.

\section{7 right hand side case-wheeler lifts $1 \mathrm{R}-7 \mathrm{R}$} with aisle-side backpack buffer

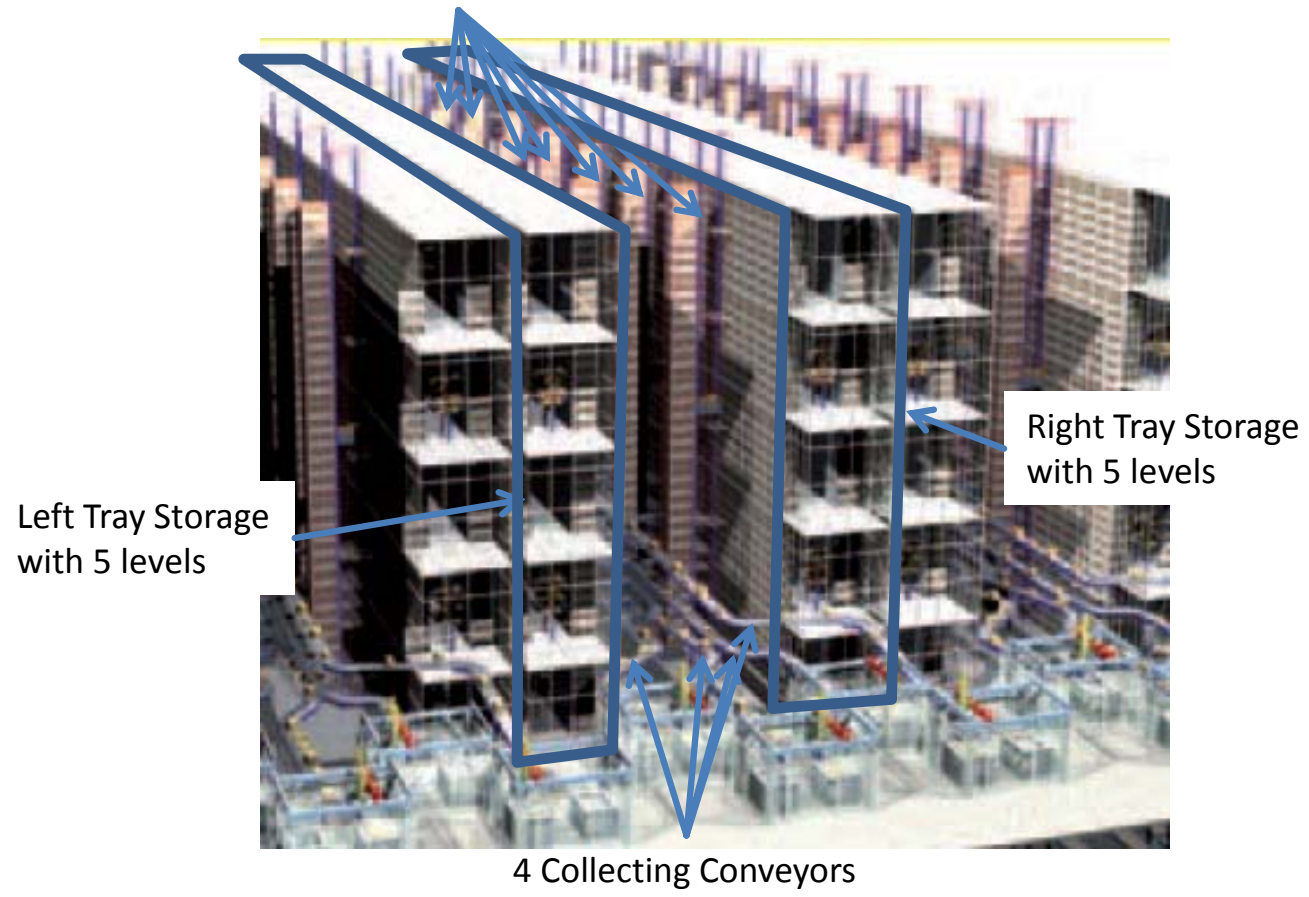

Figure 4: Schematic diagram of an SCP module with case-wheeler lifts and collecting conveyors

After having "wheeled" cases onto the intermediate buffer, the case-wheeler lift is bringing trays partially filled with cases either directly back to a transfer location from where the AS/RS devices are storing them in the STS again, or the partially filled trays are put from the lift onto a so-called backpack buffer. The backpack buffer locations are another sort of intermediate buffer location installed to have trays with a very short access time available for the case-wheeler lifts.

The first phase of simulation activities was focused around the case-wheeler lift and the collecting conveyors. For this purpose, the STS aisles were modeled in a simplified manner (without considering the $\mathrm{AS} / \mathrm{RS}$ devices and the storage locations in detail), and the automatic palletizers were working without any breakdowns. The purposes of this first phase were twofold:

- There was a rather hardware-oriented task for the simulation with respect to the speed of the casewheeler lifts, the time needed to wheel cases from a tray depending on the position of the cases 


\section{Bleifuß, Spieckermann, and Stauber}

on a tray, speed of the collecting conveyors, capacity of the intermediate buffer, and availability of these components.

- In addition to that, the simulation was used to develop the control software. Here, the focus was on the sequencing of customer orders, the sequencing of the single cases, time of retrievals from the STS, rules for the usage of the backpack puffer.

Intermediate buffers from lift to colllecting conveyors (samples)

lift $7 \mathrm{~L}$

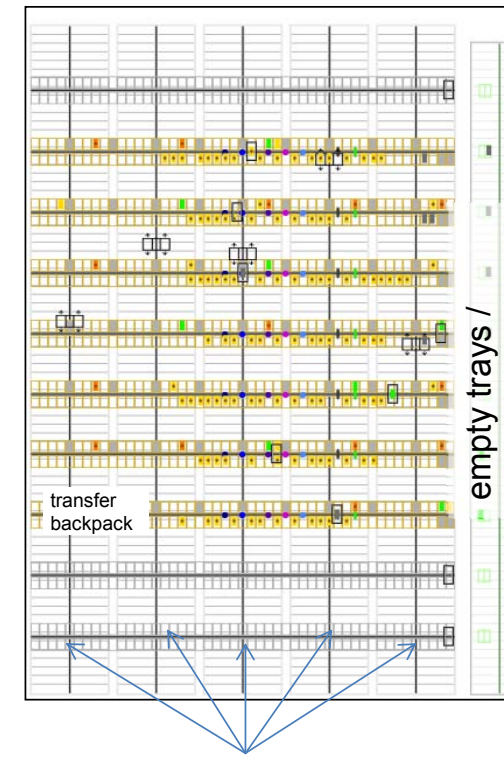

Left Tray Storage

Level 1-5

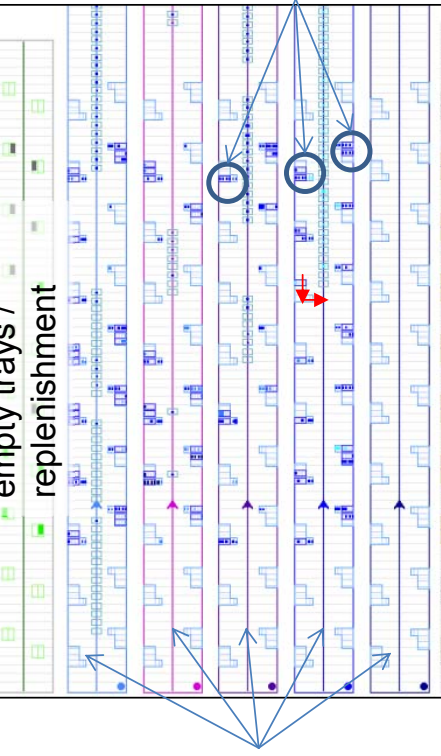

Collection Conveyors 1-5

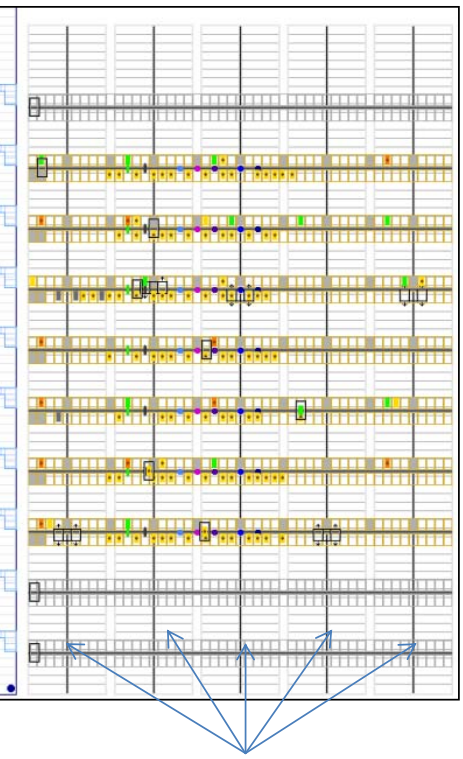

Right Tray Storage

Level 1-5 lift 7R

$\cdots$

lift $2 \mathrm{R}$

lift $1 R$

Figure 5: Schematic 2D-simulation layout of a SCP module with case-wheeler lifts and collecting conveyors

Critical issues turned out to be the number of not fully loaded trays in the STS, the transportation distance of the trays within the STS, i.e., it had to be avoided to transport the tray from, for example, a storage location near lift 1 all the way down to lift 5 for retrieval, and a balanced use of the case-wheeler lifts in the system.

With respect to the order data and the number of SKUs, different scenarios were evaluated in this phase. On the one hand, generated test cases were used and on the other hand real-world data from past SSI projects were taken. Figure 6 is giving an example on the analysis conducted in this phase. It is showing performance indicators of one SCP module during one shift, i. e., from $7.30 \mathrm{am}$ until $3.00 \mathrm{pm}$. The actual values on the y-axis had to be removed, but the purpose of the analysis can nevertheless be illustrated: the data "PALL_CASES" is indicating how the palletizer of the SCP module is performing. There are interruptions either due to pallet exchange or due to lack of supply on the collecting conveyor. In the latter case, detailed causes for missing supply of course had to be detected. One parameter impacting the palletizer performance is the data "ACTIVE_PICK_CASES" which is showing how many cases have been retrieved from the tray storage without having yet been wheeled down from a tray by a casewheeler. This might be considered to be some "pre-lift work in progress". It is obvious that a concerted balance between these two parameters is needed in order to neither congest the case-wheeler lifts nor to starve the palletizer and the simulation experiments helped to determine this balance.

However, the main benefit of the simulation was not a single output analysis, but rather learning effects with respect to the set-up of the control rule, the usage of the backpack buffer, or the ratio between the number of collection conveyors and the number of case-wheeler lifts. All in all, the simulation was used as integral part of the SCP design. 


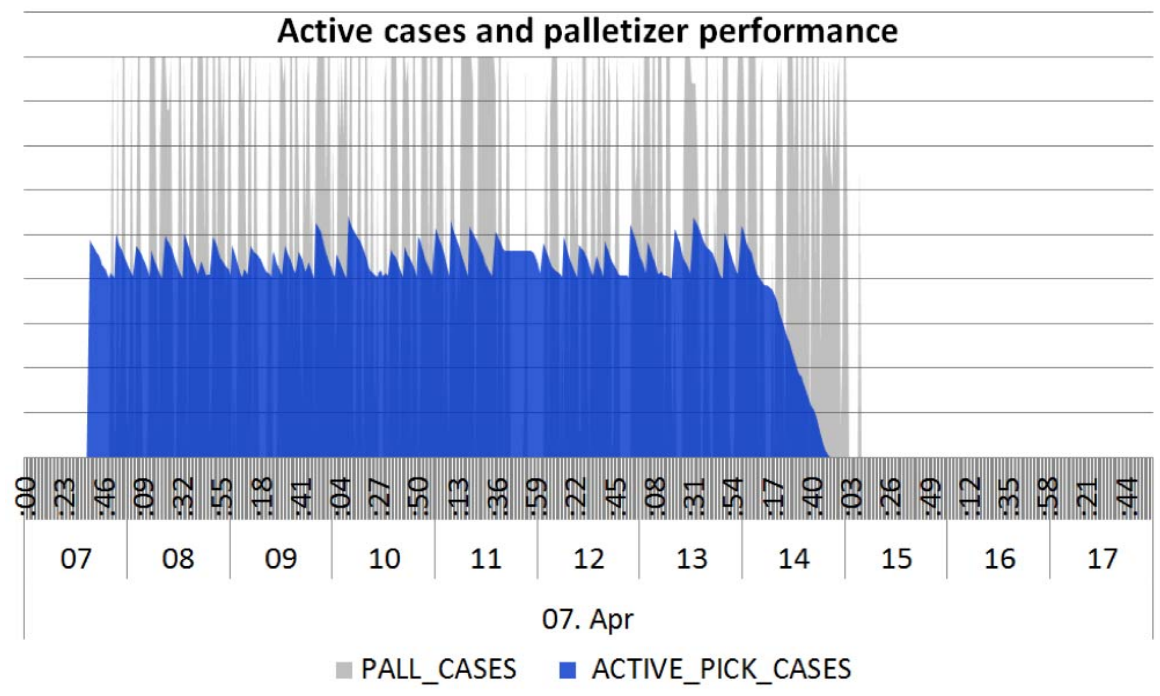

Figure 6: Released pick orders and resulting cases waiting to be palletized

\subsection{Detailed STS Simulation}

In this second phase, the SCP design simulation was extended by a detailed consideration of the tray storage. One reason for a closer look at this storage area was, that the heights of loaded trays are different, depending on the height of the stored cases as, e.g., Figure 2 and Figure 3 are illustrating. The steel construction of the STS does not preset fixed heights for the storage locations but allows for a flexible and dynamic occupation. Hence the development of storage rules was one emphasis in this phase.

Since the detailed STS simulation was integrated in the model existing from the first phase, there also were some new insights into the behavior of the lifts and the conveyors. Also, the handling of empty trays and the storage of replenishment (fully loaded trays coming from the layer tray creation) was analyzed.

\subsection{Overall SCP Concept Simulation}

Purpose of the third simulation phase was to consider the overall SCP process as depicted in Figure 3. The pallet storage and the layer tray creation were added to the model already comprising STS, lifts, and collecting conveyors. One of the issues in this phase was, whether after retrieval of a pallet from the pallet store to set one layer of cases onto a tray and store the partially loaded pallet again or to put all layers one after the other on several trays thus emptying the pallet completely. Apparently, these two alternatives have a significant impact on the balance of inventory between pallet and tray storage.

The third phase was also used to test the impact of quality checks and of so-called clearing processes, which may become necessary if the automatic processes fail for whatever reason. A failure in this case means that either a layer is not removed properly from a pallet and the trays are not filled properly or that the case-wheelers are not able to wheel cases from a tray onto the intermediate buffer.

At the end of the third phase, SSI had a new concept for a case picking system, tested in literally hundreds of different configurations in the virtual simulation environment. What happened in parallel is the construction of the components and the real-world implementation of an SCP system at a "laboratory scale" in the SSI technology center. The findings from this lab installation were continuously synchronized with the concept simulation, e.g., in order to adjust technical parameters.

\section{SIMULATION OF A SCP ADAPTATION FOR A WHOLESALER}

With the confidence gained from thorough design, virtual and real-world testing, SSI started a first implementation of the SCP at a US-based wholesaler. Again, there were three phases of simulation activi- 


\section{Bleifuß, Spieckermann, and Stauber}

ties: one phase of design simulation, an emulation during the implementation of the real-world control software and the ongoing support of the daily operation of the warehouse.

\subsection{Project Specific Simulation of SCP Design}

The main purpose of this fourth SCP simulation phase was to support the specific SCP design for the wholesaling company. It could be argued that there have been plenty of design simulations of the SCP before as elaborated in section 3. However, it needs to be pointed out that the SCP concept offers scalability in several dimensions, e.g., with respect to

- the number of STS units,

- the number of case-wheeler lifts per STS unit, and

- the number of collecting conveyors and palletizers per STS unit.

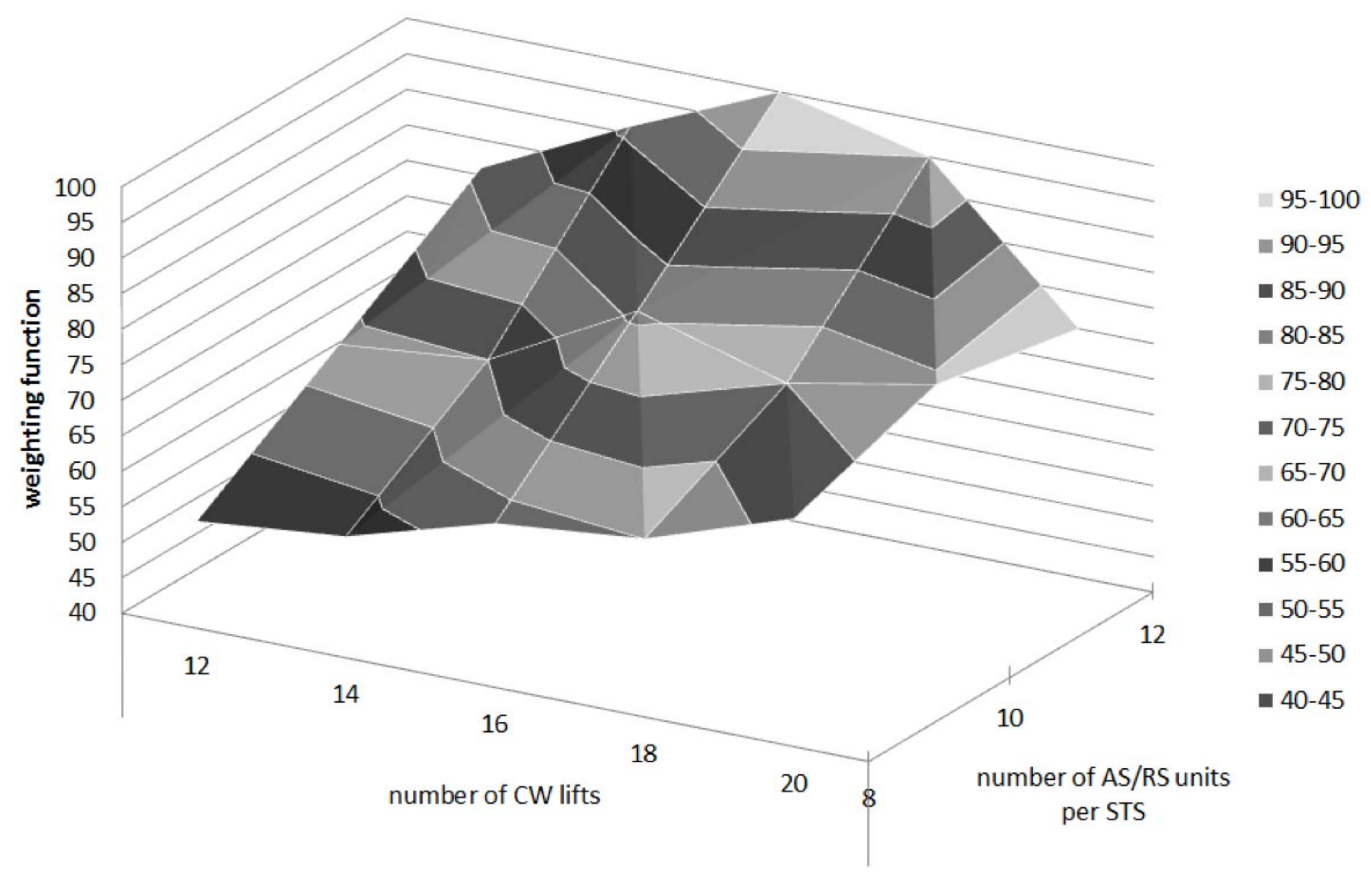

Figure 7: Overview on evaluated SCP module configurations (three collecting conveyors fixed)

And of course, the specific order structure and the number of SKUs of the wholesaler had to be used for the project specific simulations. Hence, it was decided to set up a simulation model of the STS configuration for the wholesaler which ended up with an SCP module configuration of $2 \cdot 8=16$ case-wheeler lifts, $2 \cdot 6=12 \mathrm{AS} / \mathrm{RS}$ units and three collecting conveyors and palletizers per SCP module. Figure 7 is illustrating the evaluated SCP module configurations. Again, the actual performance indicators had to be removed, however, the relative weighting function is indicating the performance of the different configurations. More than $12 \mathrm{AS} / \mathrm{RS}$ units per SCP module were not considered because of physical restrictions. The number of collecting conveyors was fixed to three which was derived from the expected number of outgoing customer pallets. The same calculation in conjunction with physical restrictions (building, space) let to the decision to set up the overall SCP for the wholesaler with three SCP modules.

In addition to the evaluation of system configurations, the simulation was also used to get a better understanding of the allocation of SKUs within the three SCP modules and of the required minimum stock, the replenishment quantities etc. 


\section{Bleifuß, Spieckermann, and Stauber}

\subsection{Emulation of MFC and WMS}

The software architecture controlling an SCP installation like the one in the distribution center of the wholesaler follows the typical levels in material handling systems: a WMS (Warehouse Management System) is communicating with the ERP (Enterprise Resource Planning) and is, e.g., exchanging customer order data or SKU related master data. While the WMS is in charge of the order management and order sequencing, administration of inventory and replenishment etc., the MFC (Material Flow Controller) is keeping track of the equipment (lifts, AR/RS devices, buffer etc.) and is managing the utilization. The PLC (Programmable Logic Controller) is (at least in the case of this implantation) responsible for the direct control of sensors, switches, electric motors etc. on shop floor level.

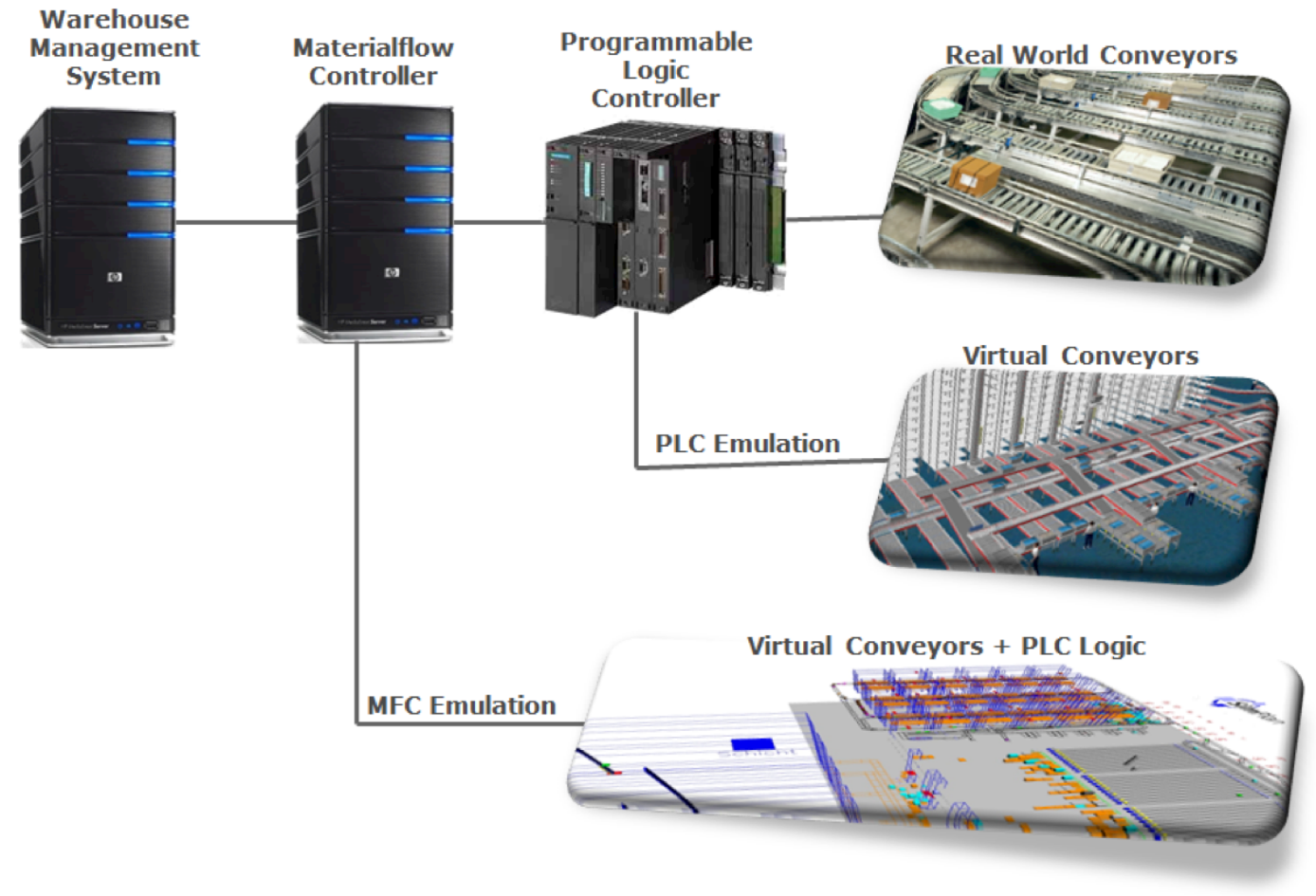

Figure 8: Schema of different emulation approaches

Purpose of an emulation environment is to test one of these control levels not in a real-world context, but by connecting a simulation ("emulation") model to the respective controller which is behaving (almost) like the real system would. In a PLC emulation, the real-world conveyor system would be replaced by an emulation model only comprising the conveyors. Whenever a conveyor needs a decision (start motor, stop motor, move pusher etc.) the emulation sends a signal to the real-world PLC, the PLC is executing its program and is sending the result back to the emulation model. When a MFC emulation is done, conveyors and PLC logic are included in the emulation model, and whenever a (emulated) PLC needs to communicate with the MFC, telegrams (in many cases TCP/IP telegrams) are exchanged. Figure 8 is illustrating the two levels of emulation.

If the architecture of the MFC and of the controls in the emulation model are carefully synchronized it is becoming possible to perform tests of subsystems of the MFC. In the SCP case with several components, one might for example want to test the real-world logic controlling the STS AS/RS devices only, while all the other MFC logic (control of lifts, conveyors, pallet storage etc.) is still simulated. The fifth phase of the project followed exactly this route, i.e., a MFC emulation environment was set up, and this environment was used to test the control of each subsystem of the SCP separately. This involved the implementation of several different types of telegrams, since a case-wheeler lift requires different control in- 


\section{Bleifuß, Spieckermann, and Stauber}

formation than a palletizing unit etc. After satisfactory emulations of the subsystems, the emulation model was tested against the overall MFC. There were tests with high volume, i.e. with the peak volume the system was designed for, as well as stress tests with about $120 \%$ of the peak volume conducted. Additionally, there were several specific test cases initiated for errors such as no-reads, non-unique barcodes in the system, unexpected states of storage locations, unexpected errors in sequences, breakdowns).

The software developers started using the emulation during software development at their premises. Later on, during ramp-up of the system at the emulation was used on-site: new releases were tested in the emulation environment and if the tests were passed, the new release was cleared for application in the real-world. Finally, the specialists of the wholesaler also used the emulation for (in the true sense of the meaning) virtual commissioning, since the time windows for tests with the real-world system were so limited, that some of the test procedures could only be performed using the emulated system.

All in all, the benefits reported from other projects as were clearly affirmed (McGregor 2002, Ramnath and Jorgensen 2012): reduced overall test effort, higher software quality, reduced ramp-up time, reduced ramp-up costs.

\subsection{Ongoing Use of the Test Environment}

The software specialists of the wholesaler decided to maintain the emulation environment implemented during ramp-up and use it on a permanent basis. The originally intended purpose of this decision was to be able to preserve the ability of doing software tests for inevitable software updates during the lifecycle of the logistics system. It turned out, however, that the virtual system was useful in other respects: in the first month of the SCP operation, the operators tended to manually intervene in the control logic. One significant example was that they would not let decide the automatic logic on the use of the backpack buffers but would decide themselves which trays to keep there. The virtual environment was used to convince them, that a dynamic automatic administration of the buffers would in fact lead to better results, even if the reason for buffering specific trays was not always immediately evident. After almost two years since start of operation, the emulation system is still used on a regular basis.

\section{SUMMARY}

The case described in this paper is an example for the use of simulation (and emulation) during several phases of a system life-cycle over several design stages all the way down to system installation and system operation (Kosturiak and Gregor 1999). It demonstrates how fruitful and intense the interaction between design and simulation engineers might be. SSI is proceeding in a similar manner in the development of other innovative material handling solutions. With respect to the presented material handling solution, the paper illustrates the level of complexity and the degree of automation in modern distribution centers. From today's point of view it appears rather likely that this trend to complexity and automation will continue during the years to come which in turn means that the demand for qualified simulation and emulation in the field will remain solid.

\section{REFERENCES}

Ashayeri, J., and L. F. Gelders. 1985. "Warehouse Design and Optimization". In European Journal of Operational Research 21:285-294.

Auinger, F., M. Vorderwinkler, and G. Buchtela. 1999. "Interface Driven Domain-Dependent Modeling Archtitecture for 'Soft-Commissioning' and 'Reality in the Loop'". In Proceedings of the 1999 Winter Simulation Conference, Edited by P. A. Farrington, H. B. Nembhard, D. T. Sturrock, and G. W. Evans, 798-805. Piscataway, New Jersey: Institute of Electrical and Electronics Engineers, Inc.

Baker, P., and M. Canessa. 2009. "Warehouse Design: A Structured Approach.” In European Journal of Operational Research 193:425-436.

Bangsow, S. 2010. Manufacturing Simulation with Plant Simulation and SimTalk. Berlin: Springer. 


\section{Bleifuß, Spieckermann, and Stauber}

Gilmore, D., and C. Holste. 2009. Automated Case Picking 2009: The Next Frontier in Distribution Center Management. Report from SupplyChainDigest and Distribution Digest, Springboro, Ohio.

Gu, J., M. Goetschalckx, and L. F. McGinnis. 2010. "Research on Warehouse Design and Performance Evaluation: A Comprehensive Review.” In European Journal of Operational Research 203:539-549.

Johnstone, M., D. Creighton, and S. Nahavandi. 2007. "Enabling industrial scale simulation / emulation models." In: Proceedings of the 2007 Winter Simulation Conference, Edited by S. G. Henderson, B. Biller, M.-H. Hsieh, J. Shortle, J. D. Tew, and R. R. Barton, 1028-1034. Piscataway, New Jersey: Institute of Electrical and Electronics Engineers, Inc.

Kosturiak, J., and M. Gregor. 1999. "Simulation in Production System Life Cycle." In Computers in Industry 38:159-172.

McGregor, I. 2002. "The Relationship between Simulation and Emulation". In Proceedings of the 2002 Winter Simulation Conference, Edited by E. Yücesan, C.-H. Chen, J. L. Snowdon, and J. M. Charnes, 1683-1688. Piscataway, New Jersey: Institute of Electrical and Electronics Engineers, Inc.

Ramnath, V., and A. Jorgensen. 2012. "Emulation: Taking Simulation Closer to Reality". In: Proceedings of the International Simulation Conference of India, http://www.ieor.iitb.ac.in/files/ieorweb/ISCI2012/F2\%20Simulation\%20Software\%20\&\%20Modelin g/ISCI2012_paper_\%2354.pdf [Accessed May 23 $\left.{ }^{\text {rd }}, 2012\right]$

Rouwenhorst, B., B. Reuter, V. Stockrahm, G. J. van Houtum, R. J. Mantel, and W. H. M. Zijm. 2000. "Warehouse Design and Control: Framework and Literature Review". In European Journal of Operational Research 122:515-533.

Roodbergen, K. J., and I. F. A. Vis. 2009. "A Survey on Literature on Automated Storage and Retrieval Systems.” In European Journal of Operational Research 194:343-362.

Smith, J. S. 2003. "Survey on the Use of Simulation for Manufacturing System Design and Operation." In Journal of Manufacturing Systems 22:157-161.

\section{AUTHOR BIOGRAPHIES}

RALF BLEIFUB is Senior Vice President at SSI Schäfer Noell GmbH, Giebelstadt, Germany, and responsible for the design and enhancement of new state-of-the-art logistics solutions within the world wide SSI Schäfer group. 1987, he started to use simulation software to design and analyze entire electronic systems used for scientific research satellites. More than 12 years he was in charge of all international software development centers from SSI Schäfer Automation group with more than 220 software engineers. He has developed together with the University of Würzburg a special emulation software for automatic warehouses and is cooperating in simulation and emulation of high complex logistics projects with Simplan. His email address is ralf.bleifuss@ssi-schaefer-noell.com.

SVEN SPIECKERMANN, Ph.D., is Chief Executive Officer at SimPlan AG, Maintal, Germany, mainly working as a senior consultant and project manager in simulation projects for several industries. Since 1992, he has been participating in over 200 simulation projects and various joint research initiatives. Additionally, he has been giving lectures in simulation at the Technical University of Braunschweig since 1995 and at the Technical University of Darmstadt since 2008. He has published several papers on simulation, simulation-based optimization and related topics. His email contact is sven.spieckermann@simplan.de.

STEPHAN STAUBER is branch manager of the SimPlan office in Regensburg, focusing on large scale simulations in logistic projects. After several years as software developer of material handling and warehouse management systems for automatic warehouses in the US, he changed to SimPlan in 1999. Since 2002 he is in charge of the SimPlan office in Regensburg. In addition to simulation and emulation projects, he has been participating in several research projects. He has been working as the SimPlan project manager in the given case. His email address is stephan.stauber@simplan.de. 